\title{
Parametric Markov Chains: PCTL Complexity and Fraction-free Gaussian Elimination*
}

\author{
Lisa Hutschenreiter Christel Baier \\ Joachim Klein \\ Technische Universität Dresden, Dresden, Germany \\ \{Lisa.Hutschenreiter, Christel.Baier, Joachim.Klein\}@tu-dresden.de
}

\begin{abstract}
Parametric Markov chains have been introduced as a model for families of stochastic systems that rely on the same graph structure, but differ in the concrete transition probabilities. The latter are specified by polynomial constraints for the parameters. Among the tasks typically addressed in the analysis of parametric Markov chains are (1) the computation of closed-form solutions for reachabilty probabilities and other quantitative measures and (2) finding symbolic representations of the set of parameter valuations for which a given temporal logical formula holds as well as (3) the decision variant of (2) that asks whether there exists a parameter valuation where a temporal logical formula holds. Our contribution to (1) is to show that existing implementations for computing rational functions for reachability probabilities or expected costs in parametric Markov chains can be improved by using fraction-free Gaussian elimination, a long-known technique for linear equation systems with parametric coefficients. Our contribution to (2) and (3) is a complexity-theoretic discussion of the model checking problem for parametric Markov chains and probabilistic computation tree logic (PCTL) formulas. We present an exponential-time algorithm for (2) and a PSPACE upper bound for (3). Moreover, we identify fragments of PCTL and subclasses of parametric Markov chains where (1) and (3) are solvable in polynomial time and establish NP-hardness for other PCTL fragments.
\end{abstract}

\section{Introduction}

Finite-state Markovian models are widely used as an operational model for the quantitative analysis of systems with probabilistic behaviour. In many cases, only estimates of the transition probabilities are available. This, for instance, applies to fault-tolerant systems where the transition probabilities are derived from error models obtained using statistical methods. Other examples are systems operating with resource-management protocols that depend on stochastic assumptions on the future workload, or cyberphysical systems where the interaction with its environment is represented stochastically. Furthermore, often the transition probabilities of Markovian models depend on configurable system parameters that can be adjusted at design-time. The task of the designer is to find a parameter setting that is optimal with respect to a given objective. This motivated the investigation of interval Markov chains (IMCs) [18] specifying intervals for the transition probabilities (rather than concrete values). More general is the model of parametric Markov chains (pMCs), which has been introduced independently by Daws [8] and Lanotte et al. [22], where the transition probabilities are given by polynomials with rational coefficients over a fixed set of real-valued parameters $x_{1}, \ldots, x_{k}$. These concepts can be further generalized to accommodate rational functions, i. e., quotients of polynomials, as transition probabilities (see, e. g., [14]).

\footnotetext{
${ }^{*}$ The authors are supported by the DFG through the Collaborative Research Center SFB 912 - HAEC, the Excellence Initiative by the German Federal and State Governments (cluster of excellence cfaed), the Research Training Group QuantLA (GRK 1763) and the DFG-projects BA-1679/11-1 and BA-1679/12-1.
}

P. Bouyer, A. Orlandini \& P. San Pietro (Eds.): 8th Symposium on Games, Automata, Logics and Formal Verification (GandALF'17) EPTCS 256, 2017, pp. 16-30 doi 10.4204/EPTCS.256.2 (c) L. Hutschenreiter, C. Baier \& J. Klein

This work is licensed under the Creative Commons Attribution-Noncommercial-Share Alike License. 
It is well-known that the probabilities $p_{s}$ for reachability conditions $\diamond$ Goal in parametric Markov chains with a finite state space $S$ can be characterized as the unique solution of a linear equation system $A \cdot p=b$ where $p=\left(p_{s}\right)_{s \in S}$ is the solution vector, and $A=A\left(x_{1}, \ldots, x_{k}\right)$ is a matrix where the coefficients are rational functions. Likewise, $b=b\left(x_{1}, \ldots, x_{k}\right)$ is a vector whose coefficients are rational functions. Note that it is no limitation to assume that the entries in $A$ and $b$ are polynomials, as rational function entries can be converted to a common denominator, which can then be removed. Now, $A \cdot p=b$ can be viewed as a linear equation system over the field $\mathbb{Q}\left(x_{1}, \ldots, x_{k}\right)$ of rational functions with rational coefficients. As a consequence, the probabilities for reachability conditions are rational functions. This has been observed independently by Daws [8] and Lanotte et al. [22] for pMCs. Daws [8] describes a computation scheme that relies on a state-elimination algorithm inspired by the state-elimination algorithm for computing regular expressions for nondeterministic finite automata. This, however, is fairly the same as Gaussian elimination for matrices over the field of rational functions.

As observed by Hahn et al. [14], the naïve implementation of Gaussian elimination for pMCs, that treats the polynomials in $A$ and $b$ as syntactic atoms, leads to a representation of the rational functions $p_{s}=p_{s}\left(x_{1}, \ldots, x_{k}\right)$ as the quotient of extremely (exponentially) large polynomials. In their implementation PARAM [13] (as well as in the re-implementation within the tool PRISM [21]), the authors of [14] use computer-algebra tools to simplify rational functions in each step of Gaussian elimination by identifying the greatest common divisor ( $\mathrm{gcd}$ ) of the numerator and the denominator polynomial. Together with polynomial-time algorithms for the gcd-computation of univariate polynomials, this approach yields a polynomial-time algorithm for computing the rational functions for reachability probabilities in pMCs with a single parameter. Unfortunately, gcd-computations are known to be expensive for the multivariate case (i. e., $k \geqslant 2$ ) [12]. To mitigate the cost of the gcd-computations, the tool Storm [10] successfully uses techniques proposed in [17] such as caching and the representation of the polynomials in partially factorized form during the elimination steps. However, it is possible to completely avoid gcd-computations by using one-step fraction-free Gaussian elimination. Surprisingly, this has not yet been investigated in the context of pMCs, although it is a well-known technique in mathematics. According to Bareiss [2], this variant of Gaussian elimination probably goes back to Camille Jordan (1838-1922), and has been rediscovered several times since. Like standard Gaussian elimination it relies on the triangulation of the matrix, and finally obtains the solution by back substitution. Applied to matrices over polynomial rings the approach generates matrices with polynomial coefficients (rather than rational functions) and ensures that the degree of the polynomials in all intermediate matrices grows at most linearly. This is achieved by dividing, in each elimination step, by a factor known by construction. Thus, when applied to a pMC with linear expressions for the transition probabilities, the degree of all polynomials in the solution vector is bounded by the number of states. For the univariate case $(k=1)$, this yields an alternative polynomial-time algorithm for the computation of the rational functions for reachability probabilities. Analogous statements hold for expectations of random variables that are computable via linear equation systems. This applies to expected accumulated weights until reaching a goal, and to the expected mean payoff.

Contribution. The purpose of the paper is to study the complexity of the model checking problem for pMCs and probabilistic computation tree logic (PCTL) [15], and its extensions by expectation operators for pMCs augmented by weights for its states. In the first part of the paper (Section 3), we discuss the use of Bareiss' one-step fraction-free Gaussian elimination for the computation of reachability probabilities. The second part of the paper (Section 4) presents complexity-theoretic results for the PCTL model checking problem in pMCs. We describe an exponential-time algorithm for computing a symbolic representation of all parameter valuations under which a given PCTL formula holds, and provide a PSPACE upper bound for 
the decision variants that ask whether a given PCTL formula holds for some or all admissible parameter valuations. The known NP-/coNP-hardness results for IMCs [26, 6] carry over to the parametric case. We strengthen this result by showing that the existential PCTL model checking problem remains NP-hard even for acyclic pMCs and PCTL formulas with a single probability operator. For the univariate case, we prove NP-completeness for the existential PCTL model checking problem, and identify two fragments of PCTL where the model checking is solvable in polynomial time. The first fragment are Boolean combinations of threshold constraints for reachability probabilities, expected accumulated weights until reaching a goal, and expected mean payoffs. The second fragment consists of PCTL formulas in positive normal form with lower probability thresholds interpreted over pMCs satisfying some monotonicity properties. Furthermore, we observe that the model checking problem for PCTL with expectation operators for reasoning about expected costs until reaching a goal is in $\mathrm{P}$ for Markov chains where the weights of the states are given as polynomials over a single parameter, when restricting to Boolean combinations of the expectation operators.

Proofs and further details on the experiments omitted in the main part due to space constraints can be found in the extended version [16].

Related work. Fraction-free Gaussian elimination is well-known in mathematics, and has been further investigated in various directions for matrices over unique factorization domains (such as polynomial rings), see e. g. [23, 19, 27, 24]. To the best of our knowledge, fraction-free Gaussian elimination has not yet been studied in the context of parametric Markovian models.

Besides the above mentioned work [8, 13, 14, 17, 9] on the computation of the rational functions for reachability probabilities in $\mathrm{pMCs}$, [22] identifies instances where the parameter synthesis problem for pMCs with 1 or 2 parameters and probabilistic reachability constraints is solvable in polynomial time. These rely on the fact that there are closed-form representations of the (complex) zero's for univariate polynomials up to degree 4 and rather strong syntactic characterizations of pMCs. In Section 3 we will provide an example to illustrate that the number of monomials in the numerators of the rational functions for reachability probabilities can grow exponentially in the number of states. We hereby reveal a flaw in [22] where the polynomial-time computability of the rational functions for reachability probabilities has been stated even for the multivariate case. [11] considers an approach for solving the parametric linear equation system obtained from sparse pMCs via Laplace expansion.

Model checking problems for IMCs and temporal logics have been studied by several authors. Most in the spirit of our work on the complexity of the PCTL model checking problem for pMCs is the paper [26] which studies the complexity of PCTL model checking in IMCs. Further complexity-theoretic results of the model checking problem for IMCs and temporal logics have been established in [6] for omega-PCTL (extending PCTL by Boolean combinations of Büchi and co-Büchi conditions), and in [5] for linear temporal logic (LTL). Our results of the second part can be seen as an extension of the work [26, 6] for the case of pMCs. The NP lower bound for the multivariate case and a single threshold constraint for reachability probabilities strengthen the NP-hardness results of [26].

There exist several approaches to obtain regions of parameter valuations of a pMC in which PCTL formulas are satisfied or not, resulting in an approximative covering of the parameter space. PARAM [14, 13] employs a heuristic, sampling based approach, while PROPhESY [9] relies on SMT solving via the existential theory of the reals to determine whether a given formula holds for all valuations in a sub region. For the same problem, [25] uses a parameter lifting technique that avoids having to solve the parametric equation system by obtaining lower and upper bounds for the values in a given region by a reduction to non-parametric Markov decision processes. 


\section{Preliminaries}

The definitions in this section require a general understanding of Markov models, standard model checking, and temporal logics. More details can be found, e. g., in [20, 1].

Discrete-time Markov chain. A (discrete-time) Markov chain (MC) $\mathscr{M}$ is a tuple $\left(S, s_{\text {init }}, E, P\right)$ where $S$ is a non-empty, finite set of states with the initial state $s_{\text {init }} \in S, E \subseteq S \times S$ is a transition relation, and $P: S \times S \rightarrow[0,1]$ is the transition probability function satisfying $P(s, t)=0$ if and only if $(s, t) \notin E$, and $\sum_{t \in S} P(s, t)=1$ for all $s \in S$ with Post $(s) \stackrel{\text { def }}{=}\{t \in S:(s, t) \in E\}$ nonempty. We refer to $G_{\mathscr{M}}=(S, E)$ as the graph of $\mathscr{M}$. A state $s \in S$ in which Post $(s)=\varnothing$ is called a trap (state) of $\mathscr{M}$.

An infinite path in $\mathscr{M}$ is an infinite sequence $s_{0} s_{1} \ldots \in S^{\omega}$ of states such that $\left(s_{i}, s_{i+1}\right) \in E$ for $i \in \mathbb{N}$. Analogously, a finite path in $\mathscr{M}$ is a finite sequence $s_{0} s_{1} \ldots s_{m} \in S^{*}$ of states in $\mathscr{M}$ such that $\left(s_{i}, s_{i+1}\right) \in E$ for $i=0,1, \ldots, m-1$. A path is called maximal if it is infinite or ends in a trap. Paths $(s)$ denotes the set of all maximal paths in $\mathscr{M}$ starting in $s$. Relying on standard techniques, every MC induces a unique probability measure $\operatorname{Pr}_{s}^{\mathscr{M}}$ on the set of all paths.

Parameters, polynomials, and rational functions. Let $x_{1}, \ldots, x_{k}$ be parameters that can assume any real value, $\bar{x}=\left(x_{1}, \ldots, x_{k}\right)$. We write $\mathbb{Q}[\bar{x}]$ for the polynomial ring over the rationals with variables $x_{1}, \ldots, x_{k}$. Each $f \in \mathbb{Q}[\bar{x}]$ can be written as a sum of monomials, i. e., $f=\sum_{\left(i_{1}, \ldots, i_{k}\right) \in I} \alpha_{i_{1}, \ldots, i_{k}} \cdot x_{1}^{i_{1}} \cdot x_{2}^{i_{2}} \cdot \ldots \cdot x_{k}^{i_{k}}$ where $I$ is a finite subset of $\mathbb{N}^{k}$ and $\alpha_{i_{1}, \ldots, i_{k}} \in \mathbb{Q}$. If $I$ is empty, or $\alpha_{i_{1}, \ldots, i_{k}}=0$ for all tuples $\left(i_{1}, \ldots, i_{k}\right) \in I$, then $f$ is the null function, generally denoted by 0 . The degree of $f$ is $\operatorname{deg}(f)=\max \left\{i_{1}+\ldots+i_{k}\right.$ : $\left.\left(i_{1}, \ldots, i_{k}\right) \in I, \alpha_{i_{1}, \ldots, i_{k}} \neq 0\right\}$ where $\max (\varnothing)=0$. A linear function is a function $f \in \mathbb{Q}[\bar{x}]$ with $\operatorname{deg}(f) \leqslant 1$. A rational function is a function of the form $f / g$ with $f, g \in \mathbb{Q}[\bar{x}], g \neq 0$. The field of all rational functions is denoted by $\mathbb{Q}(\bar{x})$. We write Constr $[\bar{x}]$ for the set of all polynomial constraints of the form $f \bowtie g$ where $f, g \in \mathbb{Q}[\bar{x}]$, and $\bowtie \in\{<, \leqslant,>, \geqslant,=\}$.

Parametric Markov chain. A (plain) parametric Markov chain on $\bar{x}, \mathrm{pMC}$ for short, is a tuple $\mathfrak{M}=\left(S, s_{i n i t}, E, \mathbf{P}\right)$ where $S, s_{i n i t}$, and $E$ are defined as for MCs, and $\mathbf{P}: S \times S \rightarrow \mathbb{Q}(\bar{x})$ is the transition probability function with $\mathbf{P}(s, t)=0$, i. e., the null function, iff $(s, t) \notin E$. Intuitively, a pMC defines the family of Markov chains arising by plugging in concrete values for the parameters. A parameter valuation $\bar{\xi}=\left(\xi_{1}, \ldots, \xi_{k}\right) \in \mathbb{R}^{k}$ is said to be admissible for $\mathfrak{M}$ if for each state $s \in S$ we have $\sum_{t \in S} P_{\bar{\xi}}(s, t)=1$ if Post $(s)$ nonempty, and $P_{\bar{\xi}}(s, t)>0$ iff $(s, t) \in E$, where $P_{\bar{\xi}}(s, t)=\mathbf{P}(s, t)(\bar{\xi})$ for all $(s, t) \in S \times S$. Let $X_{\mathfrak{M}}$, or briefly $X$, denote the set of admissible parameter valuations for $\mathfrak{M}$. Given $\bar{\xi} \in X$ the Markov chain associated with $\bar{\xi}$ is $\mathscr{M}_{\bar{\xi}}=\mathfrak{M}(\bar{\xi})=\left(S, s_{\text {init }}, E, P_{\bar{\xi}}\right)$. The semantics of the pMC $\mathfrak{M}$ is then defined as the family of Markov chains induced by admissible parameter valuations, i. e., $\llbracket \mathfrak{M} \rrbracket=\{\mathfrak{M}(\bar{\xi}): \bar{\xi} \in X\}$.

An augmented $p M C$ is a tuple $\mathfrak{M}=\left(S, s_{\text {init }}, E, \mathbf{P}, \mathfrak{C}\right)$ where $S, s_{\text {init }}, E$, and $\mathbf{P}$ are defined as for plain pMCs, and $\mathfrak{C} \subset$ Constr $[\bar{x}]$ is a finite set of polynomial constraints. A parameter valuation $\bar{\xi}$ is admissible for an augmented $\mathrm{pMC}$ if it is admissible for the induced plain $\mathrm{pMC}\left(S, s_{\text {init }}, E, \mathbf{P}\right)$, and satisfies all polynomial constraints in $\mathfrak{C}$. As for plain $\mathrm{pMC}$, we denote the set of admissible parameter valuations of an augmented pMC by $X_{\mathfrak{M}}$, or briefly $X$.

A, possibly augmented, pMC $\mathfrak{M}$ is called linear, or polynomial, if all transition probability functions and constraints are linear functions in $\bar{x}$, or polynomials in $\bar{x}$, respectively.

Interval Markov chain. An interval Markov chain (IMC) [26] can be seen as a special case of a linear augmented pMC with one parameter $x_{s, t}$ for each edge $(s, t) \in E$, and linear constraints $\alpha_{s, t} \unlhd_{1} x_{s, t} \unlhd_{2} \beta_{s, t}$ for each edge with $\alpha_{s, t}, \beta_{s, t} \in \mathbb{Q} \cap[0,1]$ and $\unlhd_{1}, \unlhd_{2} \in\{<, \leqslant\}$. According to the terminology introduced in [26], this corresponds to the semantics of IMC as an "uncertain Markov chain". The alternative semantics 
of IMC as a Markov decision process will not be considered in this paper.

Labellings and weights. Each of these types of Markov chain, whether MC, plain or augmented pMC, or IMC, can be equipped with a labelling function $\mathscr{L}: S \rightarrow 2^{\mathrm{AP}}$, where AP is a finite set of atomic propositions. If not explicitly stated, we assume the implicit labelling of the Markov chain defined by using the state names as atomic propositions and assigning each name to the respective state. Furthermore, we can extend any Markov chain with a weight function wgt: $S \rightarrow \mathbb{Q}$. The value assigned to a specific state $s \in S$ is called the weight of $s$. It is sometimes also referred to as the reward of $s$. In addition to assigning rational values we also consider parametric weight functions wgt: $S \rightarrow \mathbb{Q}(\bar{x})$.

Probabilistic computation tree logic. We augment the standard notion of probabilistic computation tree logic with operators for the expected accumulated weight and mean payoff, and for comparison. Let AP be a finite set of atomic propositions. $\bowtie$ stands for $\leqslant, \geqslant,<,>$, or $=, c \in[0,1], r \in \mathbb{Q}$. Then

$$
\begin{aligned}
& \Phi::=\text { true }|a| \Phi \wedge \Phi|\neg \Phi| \mathbb{P}_{\bowtie c}(\varphi)\left|\mathbb{E}_{\bowtie r}(\rho)\right| \mathbb{C}_{\operatorname{Pr}}(\varphi, \bowtie, \varphi) \mid \mathbb{C}_{\mathrm{E}}(\rho, \bowtie, \rho) \text { state formula } \\
& \varphi \quad:=\bigcirc \Phi \mid \Phi \cup \Phi \text { pathformula } \quad \rho::=\forall \Phi \mid \operatorname{mp}(\Phi) \quad \text { terms for random variables }
\end{aligned}
$$

where $a \in$ AP. The basic temporal modalities are $\bigcirc$ (next) and $\mathrm{U}$ (until). The usual derived temporal modalities $\diamond$ (eventually), R (release) and $\square$ (always) are defined by $\diamond \Phi \stackrel{\text { def }}{=}$ true $U \Phi$, and $\mathbb{P}_{\bowtie c}\left(\Phi_{1} R \Phi_{2}\right) \stackrel{\text { def }}{=}$ $\mathbb{P}_{\bowtie 1-c}\left(\left(\neg \Phi_{1}\right) \cup\left(\neg \Phi_{2}\right)\right)$, where, e.g., $\leqslant$ is $\geqslant$ and $\overline{\text { is }}>$, and $\square \Phi \stackrel{\text { def }}{=}$ false $\mathrm{R} \Phi$.

For an MC $\mathscr{M}$ with states labelled by $\mathscr{L}: S \rightarrow$ AP we use the standard semantics. We only state the semantics of the probability, expectation, and comparison operators here. For each state $s \in S, s \models \mathscr{M}$ $\mathbb{P}_{\bowtie c}(\varphi)$ iff $\operatorname{Pr}_{s}^{\mathscr{M}}(\varphi) \bowtie c$, and $s \models \mathscr{M} \mathbb{C}_{\operatorname{Pr}}\left(\varphi_{1}, \bowtie, \varphi_{2}\right)$ iff $\operatorname{Pr}_{s}^{\mathscr{M}}\left(\varphi_{1}\right) \bowtie \operatorname{Pr}_{s}^{\mathscr{M}}\left(\varphi_{2}\right)$. Here $\operatorname{Pr}_{s}^{\mathscr{M}}(\varphi)$ is short for $\operatorname{Pr}_{s}^{\mathscr{M}}\{\pi \in \operatorname{Paths}(s): \pi \models \mathscr{M} \varphi\}$. Furthermore, $s \models \mathscr{M} \mathbb{E}_{\bowtie r}(\rho)$ iff $\mathrm{E}_{s}^{\mathscr{M}}\left(\rho^{\mathscr{M}}\right) \bowtie r$, and $s \models \mathscr{M} \mathbb{C}_{\mathrm{E}}\left(\rho_{1}, \bowtie, \rho_{2}\right)$ iff $\mathrm{E}_{s}^{\mathscr{M}}\left(\rho_{1}^{\mathscr{M}}\right) \bowtie \mathrm{E}_{s}^{\mathscr{M}}\left(\rho_{2}^{\mathscr{M}}\right)$, where $\mathrm{E}_{s}^{\mathscr{M}}(\cdot)$ denotes the expected value of the respective random variable. For detailed semantics of the expectation operators, see [16]. We write $\mathscr{M}=\Phi$ iff $s_{\text {init }}=\mathscr{M} \Phi$.

Notation: PCTL+EC and sublogics. We use PCTL to refer to unaugmented probabilistic computation tree logic. If we add only the expectation operator we write PCTL+E, and, analogously, PCTL+C if we only add the comparison operator for probabilities. PCTL+EC denotes the full logic defined above.

DAG-representation and length of formulas. We consider for any PCTL+EC state formula the directed acyclic graph (DAG) representing its syntactic structure. Each node of the DAG represents one of the sub-state formulas. The use of a DAG rather than the syntax tree allows the representation of subformulas that occur several times in the formula $\Phi$ by a single node. The leaves of the DAG can be the Boolean constant true, and atomic propositions. The inner nodes of the DAG, e.g., of a PCTL formula, are labelled with one of the operators $\wedge, \neg, \mathbb{P}_{\bowtie c}(\cdot U \cdot), \mathbb{P}_{\bowtie c}(\bigcirc \cdot)$. Nodes labelled with $\neg$ and $\mathbb{P}_{\bowtie c}(\bigcirc \cdot)$ have a single outgoing edge, while nodes labelled with $\wedge$ or $\mathbb{P}_{\bowtie c}(\cdot U \cdot)$ have two outgoing edges. For the above-mentioned extensions of PCTL the set of possible inner node labels is extended accordingly. For example, a node $v$ representing the PCTL+C formula $\mathbb{C}_{\operatorname{Pr}}\left(\bigcirc \Phi_{1}, \bowtie, \Phi_{2} \cup \Phi_{3}\right)$ has three outgoing edges. If $\Phi_{1}=\Phi_{2}$ then there are two parallel edges from $v$ to a node representing $\Phi_{1}$. The length of a PCTL+EC formula is defined as the number of nodes in its DAG.

\section{Fraction-free Gaussian elimination}

Given a pMC $\mathfrak{M}$ as in Section 2, the probabilities $\operatorname{Pr}_{s}^{\mathfrak{M}(\bar{x})}(\diamond a)$ for reachability conditions are rational functions and computable via Gaussian elimination. As stated in the introduction, this has been originally observed in [8, 22] and realized, e. g., in the tools PARAM [13] and Storm [9, 10] together with techniques 


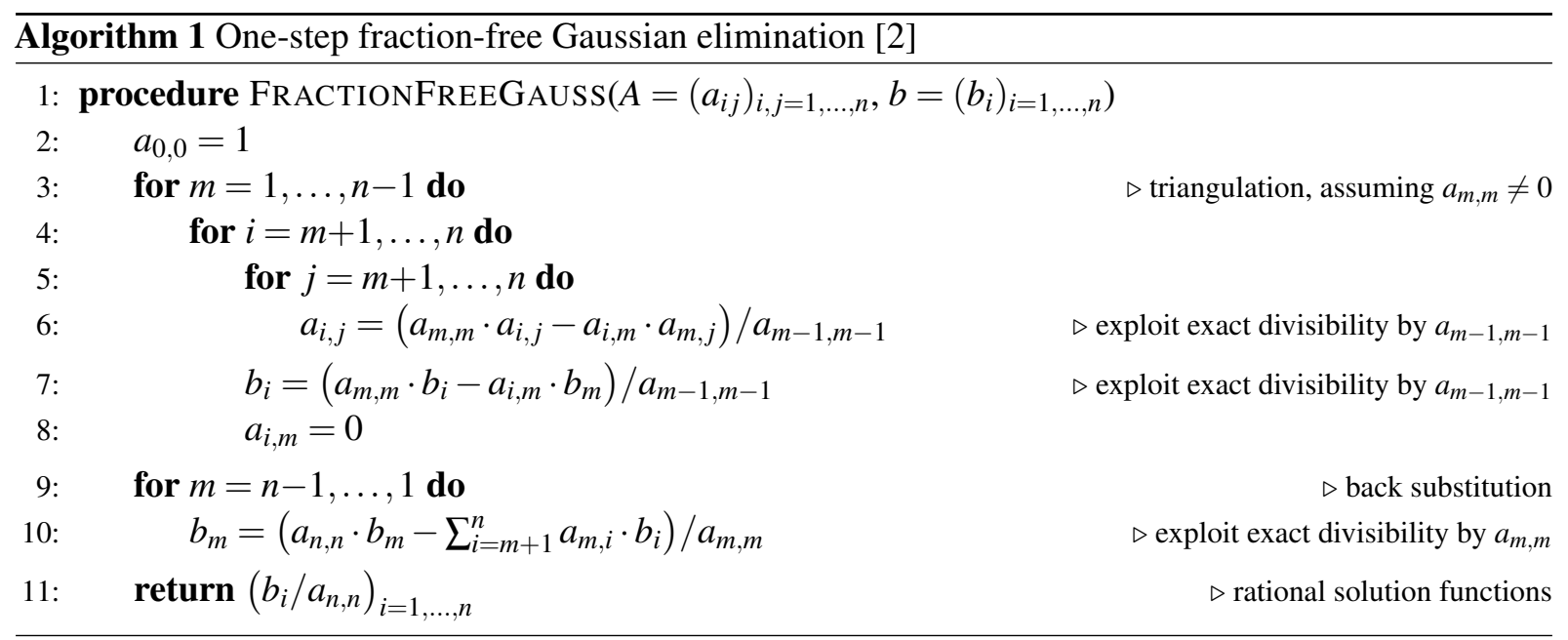

based on gcd-computations on multivariate polynomials. In this section, we discuss the potential of fraction-free Gaussian elimination as an alternative, which is well-known in mathematics [2, 12], but to the best of our knowledge, has not yet been considered in the context of pMCs.

While the given definitions allow for rational functions in the transition probability functions of (augmented) pMCs, we will focus on polynomial (augmented) pMCs throughout the remainder of the paper. Generally, a linear equation systems containing rational functions as coefficients can be rearranged to one containing only polynomials by multiplying each line with the common denominator of the respective rational functions. Due to the multiplications this involves the risk of a blow-up in the coefficient size. To avoid this we add variables in the following way. Let $\mathfrak{M}=\left(S, s_{\text {init }}, E, \mathbf{P}, \mathfrak{C}\right)$ be an (augmented) pMC. For all $(s, t) \in E$ introduce a fresh variable $x_{s, t}$. By definition $\mathbf{P}(s, t)=\frac{f_{s, t}}{s_{s, t}}$ for some $f_{s, t}, g_{s, t} \in \mathbb{Q}[\bar{x}]$. Let $\mathbf{P}^{\prime}(s, t)=f_{s, t} \cdot x_{s, t}$ if $(s, t) \in E, \mathbf{P}^{\prime}(s, t)=0$ if $(s, t) \notin E, \mathfrak{C}^{\prime}=\mathfrak{C} \cup\left\{g_{s, t} \cdot x_{s, t}=1:(s, t) \in E\right\}$. Then $\mathfrak{M}^{\prime}=\left(S, s_{\text {init }}, E, \mathbf{P}^{\prime}, \mathfrak{C}^{\prime}\right)$ is a polynomial augmented $\mathrm{pMC}$.

Linear equation systems with polynomial coefficients. Let $x_{1}, \ldots, x_{k}$ be parameters, $\bar{x}=\left(x_{1}, \ldots, x_{k}\right)$. We consider linear equation systems of the form $A \cdot p=b$, where $A=\left(a_{i, j}\right)_{i, j=1, \ldots, n}$ is a non-singular $n \times n$ matrix with $a_{i, j}=a_{i, j}(\bar{x}) \in \mathbb{Q}[\bar{x}]$. Likewise, $b=\left(b_{i}\right)_{i=1, \ldots, n}$ is a vector of length $n$ with $b_{i}=b_{i}(\bar{x}) \in \mathbb{Q}[\bar{x}]$. The solution vector $p=\left(p_{i}\right)_{i=1, \ldots, n}$ is a vector of rational functions $p_{i}=f_{i} / g_{i}$ with $f_{i}, g_{i} \in \mathbb{Q}[\bar{x}]$. By Cramer's rule we obtain $p_{i}=\frac{\operatorname{det}\left(A_{i}\right)}{\operatorname{det}(A)}$, where $\operatorname{det}(A)$ is the determinant of $A$, and $\operatorname{det}\left(A_{i}\right)$ the $\operatorname{determinant}$ of the matrix obtained when substituting the $i$-th column of $A$ by $b$. If the coefficients of $A$ and $b$ have at most degree $d$, the Leibniz formula implies that $f_{i}$ and $g_{i}$ have at most degree $n \cdot d$.

- Lemma 1. There is a family $\left(\mathfrak{M}_{k}\right)_{k \geqslant 2}$ of acyclic linear pMCs where $\mathfrak{M}_{k}$ has $k$ parameters and $n=k+3$ states, including distinguished states $s_{0}$ and goal, such that $\operatorname{Pr}_{s_{0}}^{\mathfrak{M}(\bar{x})}(\diamond$ goal $)$ is a polynomial for which even the shortest sum-of-monomial representation has $2^{k}$ monomials.

One-step fraction-free Gaussian elimination is a variant of fraction-free Gaussian elimination that allows for divisions which are known to be exact at the respective point in the algorithm. When using naïve fraction-free Gaussian elimination the new coefficients after the $m$-th step, $m=1, \ldots, n-1$, are computed as $a_{i, j}^{(m)}=a_{i, j}^{(m-1)} a_{m, m}^{(m-1)}-a_{i, m}^{(m-1)} a_{m, j}^{(m-1)}$ for $i, j=m+1, \ldots, n$, where $a_{i, j}^{(0)}=a_{i, j}$. When applied to systems with polynomial coefficients this results in the degree doubling after each step, so the degree grows exponentially. In a step of one-step fraction-free Gaussian elimination (see Algorithm 1), the computation of the coefficients changes to $a_{i, j}^{(m)}=\left(a_{i, j}^{(m-1)} a_{m, m}^{(m-1)}-a_{i, m}^{(m-1)} a_{m, j}^{(m-1)}\right) / a_{m-1, m-1}^{(m-1)}$ with $a_{0,0}^{(0)}=1$. 
Using Sylvester's identity one can prove that $a_{i, j}^{(m)}$ is again a polynomial, and that $a_{m-1, m-1}^{(m-1)}$ is in general the maximal possible divisor. The $b_{i}$ are updated analogously. If the maximal degree of the initial coefficients of $A$ and $b$ is $d$, this technique therefore guarantees, that after $m$ steps the degree of the coefficients is at most $(m+1) \cdot d$, i. e., it grows linear in $d$ during the procedure. For polynomials the division by $a_{m-1, m-1}^{(m-1)}$ can be done using standard polynomial division. The time-complexity of the exact multivariate polynomial division in this case is in each step $\mathscr{O}\left(\operatorname{poly}(m d)^{k}\right)$, so for the full one-step fraction-free Gaussian elimination it is $\mathscr{O}\left(\operatorname{poly}(n, d)^{k}\right)$.

Proposition 4.3 in [22] states that the rational functions $p_{i}=f_{i} / g_{i}$ for reachability probabilities in pMC with a representation of the polynomials $f_{i}, g_{i}$ as sums of monomials (called normal form in [22]) are computable in polynomial time. This contradicts Lemma 1 which shows that the number of monomials in the representation of a reachability probability as a sum of monomials can be exponential in the number of parameters. However, the statement is correct for the univariate case.

- Lemma 2. Let $\mathfrak{M}$ be a polynomial pMC over a single parameter and $T$ a set of states. Then, the rational functions for the reachability probabilities $\operatorname{Pr}_{s}^{\mathfrak{M}}(\diamond T)$ are computable in polynomial time. Analogously, rational functions for the expected accumulated weight until reaching $T$ or the expected mean payoff are computable in polynomial time.

Note that the degrees of the polynomials $a_{i, j}^{(m)}$ and $b_{j}^{(m)}$ computed by one-step fraction-free Gaussian elimination for reachability probabilities are bounded by $(m+1) \cdot d$, where $d=\max _{s, t \in S} \operatorname{deg}(\mathbf{P}(s, t))$, so the polynomials have representations as sums of at most $m d+1$ monomials. In particular, the degree and representation size of the final polynomials $f_{s}=b_{s}^{(n)}$ and $g_{s}=a_{s, s}^{(n)}$ for the rational functions $\operatorname{Pr}_{s}^{\mathfrak{M}(x)}(\nabla$ goal $)=f_{s} / g_{s}$ is in $\mathscr{O}(n d)$ where $n$ is the number of states of $\mathfrak{M}$. Another observation concerns the case where only the right-hand side of the linear equation system is parametric. Systems of this form occur, e. g., when considering expectation properties for MCs with parametric weights.

- Lemma 3. Let $A \cdot p=b$ be a parametric linear equation system as defined above where $A$ is parameterfree. Then the solution vector $p=\left(p_{i}\right)_{i=1, \ldots, n}$ consist of polynomials of the form $p_{i}=\sum_{i=1}^{n} \beta_{i} \cdot b_{i}$ with $\beta_{i} \in \mathbb{Q}$ and can be computed in polynomial time.

Stratification via SCC-decomposition. It is well known (e. g., [7, 17]) that for probabilistic/parametric model checking a decomposition into strongly-connected components (SCCs) can yield significant performance benefits due to the structure of the underlying models. We have adapted the one-step fraction-free Gaussian elimination approach by a preprocessing step that permutes the matrix according to the topological ordering of the SCCs. This results in the coefficient matrix already having a stair-like form at the start of the algorithm. In the triangulation part of the algorithm, each SCC can now be considered separately, as non-zero entries below the main diagonal only occur within each SCC. While the back-substitution in the general one-step fraction-free elimination will result in each entry on the main diagonal being equal to the last, this property is now only maintained within the SCCs. Formally, this means that the back substitution step in Algorithm 1 is replaced by the following:

$$
b_{m}=\left(a^{*}(\text { current SCC }) \cdot b_{m}-\sum_{i=m+1}^{n} a_{m, i} \cdot b_{i} \cdot \frac{a^{*}(\text { current SCC })}{a^{*}(\mathrm{SCC} \text { at } i)}\right) / a_{m, m}
$$

where $a^{*}(\operatorname{SCC}$ at $n)=a_{n, n}$, and, for $i=1, \ldots, n-1, a^{*}(\operatorname{SCC}$ at $i)=a^{*}(\mathrm{SCC}$ at $i+1)$ if the $i$-th and $(i+1)$-st state belong to the same SCC and $a^{*}(\mathrm{SCC}$ at $i)=a_{i, i} \cdot a^{*}(\mathrm{SCC}$ at $i+1)$ otherwise. Intuitively, $a^{*}(\mathrm{SCC}$ at $i)$ is the product of the $a$ 's on the diagonal corresponding to the last states in the current SCC and the SCCs below. Of course, the return statement also has to be adjusted accordingly. The advantage of 
Table 1: Statistics for "complete pMC". Matrix rows and number of distinct parameters, as well as time for solving the parametric equation system per solver. For $n=7$, all solvers timed out (30min).

\begin{tabular}{rrr|rrr|r}
$n$ & rows & param. & eigen & state-elim & GE-ff & red $(G E-f f)$ \\
\hline 4 & 4 & 20 & $0.47 \mathrm{~s}$ & $0.64 \mathrm{~s}$ & $0.06 \mathrm{~s}$ & $0.01 \mathrm{~s}$ \\
5 & 5 & 30 & $44.47 \mathrm{~s}$ & $42.09 \mathrm{~s}$ & $2.13 \mathrm{~s}$ & $1.52 \mathrm{~s}$ \\
6 & 6 & 42 & time-out & time-out & $221.27 \mathrm{~s}$ & $21.53 \mathrm{~s}$ \\
\hline
\end{tabular}

this approach is that the polynomials in the rational functions aside from the ones in the first strongly connected component will have an even lower degree.

Implementation and experiments. For a first experimental evaluation of the one-step fraction-free Gaussian elimination approach $(G E-f f)$ in the context of probabilistic model checking, we have implemented this method (including the SCC decomposition and topological ordering described above) as an alternative solver for parametric linear equation systems in the state-of-the-art probabilistic model checker Storm [10]. We compare GE-ff against the two solvers provided by Storm (v1.0.1) for solving parametric equation systems, i. e., the solver based on the eigen linear algebra library 11 and on state elimination (state-elim) [14]. Both of Storm's solvers use partially factorized representations of the rational functions provided by the CArL library ${ }^{2}$ This approach, together with caching, was shown [17] to be beneficial due to improved performance of the gcd-computations during the simplification steps.

It should be noted that our implementation is intended to provide first results that allow to gauge whether the fraction-free method, by avoiding gcd-computations, can be beneficial in practice and is thus rather naïve in certain aspects. As an example, it currently relies on a dense matrix representation, with performance improvements for larger models to be expected from switching to sparse representations as used in Storm's eigen and state-elim solvers. In addition to the fraction-free approach, our solver can also be instantiated to perform a straight-forward Gaussian elimination, using any of the representations for rational functions provided by the CArL library. In all our experiments, we have compared the solutions obtained by the different solvers and verified that they are the same.

Experimental studies. The source code of our extension of Storm and the artifacts of the experiments are available online $3^{3}$ As our GE-ff implementation is embedded as an alternative solver in Storm, we mainly report the time actually spent for solving the parametric equation system, as the other parts of model checking (model building, precomputations) are independent of the chosen solver. For benchmarking, we used a machine with two Intel Xeon E5-2680 8-core CPUs at 2.70GHz and with 384GB RAM, a time out of 30 minutes and a memory limit of $30 \mathrm{~GB}$. All the considered solvers run single-threaded. We have considered three different classes of case studies for experiments.

Complete pMC. As a first experiment to gauge the efficiency in the presence of a high ratio of parameters to states, we considered a family of pMCs with a complete graph structure (over $n$ states) and one parameter per transition, resulting in $n \cdot(n+1)$ parameters (for details see [16]).

Table 1 depicts statistics for the corresponding computations, for the two standard solvers in Storm (eigen and state-elim), as well as our fraction-free implementation $(G E-f f)$. For state-elim, we always use the default elimination order (forward). The time for $G E$ - $f f$ corresponds to the time until a solution rational function (for all states) is obtained. As the numerator and denominator of these rational functions

\footnotetext{
1 http://eigen.tuxfamily.org/

${ }^{2}$ https://github.com/smtrat/carl

$3 \overline{\text { http://wwwtcs.inf.tu-dresden.de/ALGI/PUB/GandALF17/ }}$
} 
Table 2: Statistics for "Israeli-Jalfon", with strong bisimulation quotienting. Matrix rows and number of distinct parameters, as well as time for solving the parametric equation system per solver.

\begin{tabular}{rrrr|rrrr|r}
$n$ & $k$ & rows & param. & eigen & state-elim & GE-fac & GE-ff & red(GE-ff) \\
\hline 4 & 3 & 21 & 4 & $1.01 \mathrm{~s}$ & $0.86 \mathrm{~s}$ & $0.73 \mathrm{~s}$ & $0.16 \mathrm{~s}$ & $0.20 \mathrm{~s}$ \\
4 & 4 & 15 & 4 & $0.94 \mathrm{~s}$ & $0.58 \mathrm{~s}$ & $0.58 \mathrm{~s}$ & $0.16 \mathrm{~s}$ & $0.13 \mathrm{~s}$ \\
5 & 2 & 16 & 5 & $19.13 \mathrm{~s}$ & $30.83 \mathrm{~s}$ & $29.46 \mathrm{~s}$ & $9.36 \mathrm{~s}$ & $0.33 \mathrm{~s}$ \\
5 & 3 & 36 & 5 & $360.43 \mathrm{~s}$ & $747.32 \mathrm{~s}$ & $172.16 \mathrm{~s}$ & $485.78 \mathrm{~s}$ & $95.92 \mathrm{~s}$ \\
5 & 4 & 51 & 5 & $457.55 \mathrm{~s}$ & $1542.97 \mathrm{~s}$ & $442.80 \mathrm{~s}$ & $614.01 \mathrm{~s}$ & $742.69 \mathrm{~s}$ \\
5 & 5 & 31 & 5 & $368.70 \mathrm{~s}$ & $1597.29 \mathrm{~s}$ & $252.92 \mathrm{~s}$ & $622.00 \mathrm{~s}$ & $414.72 \mathrm{~s}$ \\
\hline
\end{tabular}

are not necessarily coprime, for comparison purposes we list as well the time needed for simplification $(\operatorname{red}(G E-f f))$ via division by the gcd. As can be seen, here, the fraction-free approach significantly outperforms Storm's standard solvers and scales to a higher number of parameters. We confirmed using profiling that the standard solvers indeed spend most of the time in gcd-computations.

Multi-parameter Israeli-Jalfon self-stabilizing. The benchmarks used to evaluate parametric model checking implementations in previous papers tend to be scalable in the number of components but use a fixed number of parameters, usually 2. To allow further experiments with an increasing number of parameters, we considered a pMC-variant of the Israeli-Jalfon self-stabilizing protocol with $n$ processes, $k$ initial tokens and $n$ parameters (for details see [16]).

Table 2 depicts the time spent for computing the rational functions for several instances. As can be seen, the fraction-free approach is competitive for the smaller instances, with performance between the eigen and state-elim solvers for the larger instances. We have also included running times for $G E$-fac, i. e., for our naïve implementation of Gaussian elimination using the representation for rational functions as used by Storm for the standard solvers, including automatic gcd-based simplification after each step to ensure that numerator and denominator are coprime. $G E-f a c$ operates on the same, topologically sorted matrix as the fraction-free GE-ff. Curiously, GE-fac is able to outperform the eigen solver for some of the larger instances. We believe this is mainly due to differences in the matrix permutation and their effect on the elimination order, which is known to have a large impact on performance (e. g., [9]).

Benchmark case studies from [9]. Furthermore, we considered several case study instances that were used in [9] to benchmark parametric model checkers, namely the brp, crowds, egl, nand, zeroconf models. Table 3 depicts statistics for selected instances, for further details see [16]. The application of bisimulation quotienting often has a large impact on the size of the linear equation system, so we performed experiments with and without quotienting. For crowds, bisimulation quotienting was particularly effective, with all considered instances having a very small state space and negligible solving times. For the non-quotiented instances, Storm's standard solvers outperform GE-ff. For the zeroconf instance in Table 3, GE-ff is competitive. Note that the models in the brp, egl and nand case studies are acyclic and that the parametric transition probabilities and rewards are polynomial. As a consequence, the gcd-computations used in Storm's solvers don't impose a significant overhead as the rational functions during the computation all have denominator polynomials of degree zero.

Overall, the experiments have shown that there are instances where the fraction-free approach can indeed have a positive impact on performance. Keeping in mind that our implementation has not yet been significantly optimized, we believe that the fraction-free approach is an interesting addition to the gcd-based solver approaches. In particular, the application of better heuristics for the order of processing (i. e., the permutation of the matrix) could still lead to significant performance increases. 
Table 3: Selected statistics for the benchmarks of [9]. Matrix rows and number of distinct parameters, as well as time for solving the parametric equation system per solver.

\begin{tabular}{rrr|rrr|r} 
model & rows & param. & eigen & state-elim & GE-ff & red $(G E-f f)$ \\
\hline Crowds (3,5), weak-bisim & 40 & 2 & $0.08 \mathrm{~s}$ & $0.06 \mathrm{~s}$ & $0.02 \mathrm{~s}$ & $0.13 \mathrm{~s}$ \\
Crowds (5,5), weak-bisim & 40 & 2 & $0.08 \mathrm{~s}$ & $0.06 \mathrm{~s}$ & $0.02 \mathrm{~s}$ & $0.11 \mathrm{~s}$ \\
Crowds (10,5), weak-bisim & 40 & 2 & $0.08 \mathrm{~s}$ & $0.06 \mathrm{~s}$ & $0.02 \mathrm{~s}$ & $0.11 \mathrm{~s}$ \\
Crowds (3,5) & 715 & 2 & $0.99 \mathrm{~s}$ & $0.80 \mathrm{~s}$ & $11.44 \mathrm{~s}$ & $63.39 \mathrm{~s}$ \\
Crowds (5,5) & 2928 & 2 & $6.36 \mathrm{~s}$ & $5.51 \mathrm{~s}$ & $1271.95 \mathrm{~s}$ & time-out \\
Crowds (10,5) & 25103 & 2 & $139.82 \mathrm{~s}$ & $173.15 \mathrm{~s}$ & time-out & - \\
\hline Zeroconf $(1000)$ & 1002 & 2 & $81.03 \mathrm{~s}$ & $45.01 \mathrm{~s}$ & $49.43 \mathrm{~s}$ & $11.35 \mathrm{~s}$ \\
\hline
\end{tabular}

\section{Complexity of the PCTL+EC model checking problem}

We now study the complexity of the following variants of the PCTL+EC model checking problem. Given an augmented $\mathrm{pMC} \mathfrak{M}=\left(S, s_{\text {init }}, E, \mathbf{P}, \mathfrak{C}\right)$ and a PCTL+EC (state) formula $\Phi$ :

(All) Compute a representation of the set of all satisfying parameter valuations, i. e., the set of all admissible parameter valuations $\bar{\xi} \in X$ such that $\mathfrak{M}(\bar{\xi}) \models \Phi$.

(MC-E) Does there exist a valuation $\bar{\xi} \in X$ such that $\mathfrak{M}(\bar{\xi}) \models \Phi$ ?

(MC-U) Does $\mathfrak{M}(\bar{\xi}) \models \Phi$ hold for all admissible valuations $\bar{\xi} \in X$ ?

(MC-E) and (MC-U) are essentially duals of each other. Note that the answer for the universal variant (MC-U) is obtained by the negation of the answer for (MC-E) with formula $\neg \Phi$, and vice versa. In what follows, we shall concentrate on (All) and the existential model checking problem (MC-E).

Computing all satisfying parameter valuations. As before, $X=X_{\mathfrak{M}}$ denotes the set of admissible valuations. In what follows, let $\chi$ be the conjunction of the polynomial constraints in $\mathfrak{C}$ as well as the constraints $\sum_{t \in S} \mathbf{P}(s, t)=1$ for each non-trap state $s \in S$, and $0<\mathbf{P}(s, t)$ for each edge $(s, t) \in E$. We then have $\bar{\xi}=\chi$ if and only if $\bar{\xi}$ is admissible, i. e., $\bar{\xi} \in X$.

Let $\Phi$ be a PCTL+EC formula. The satisfaction function $\operatorname{Sat}_{\mathfrak{M}}(\Phi): X \rightarrow 2^{S}$ is defined by:

$$
\operatorname{Sat}_{\mathfrak{M}}(\Phi)(\bar{\xi}) \stackrel{\text { def }}{=}\left\{s \in S: s=_{\mathfrak{M}(\bar{\xi})} \Phi\right\}=\operatorname{Sat}_{\mathfrak{M}(\bar{\xi})}(\Phi)
$$

We now present an algorithm to compute a symbolic representation of the satisfaction function that groups valuations with the same corresponding satisfaction set together. More precisely, we deal with a representation of the satisfaction function $\operatorname{Sat}_{\mathfrak{M}}(\Phi)$ by a finite set $\operatorname{Sat}_{\mathfrak{M}}(\Phi)$ of pairs $(\gamma, T)$ where $\gamma$ is a Boolean combination of constraints and $T \subseteq S$ such that (i) $(\gamma, T) \in \operatorname{Sat}_{\mathfrak{M}}(\Phi)$ and $\bar{\xi} \models \gamma$ implies $T=\operatorname{Sat}_{\mathfrak{M}}(\Phi)(\bar{\xi})$, and (ii) whenever $T=\operatorname{Sat}_{\mathfrak{M}}(\bar{\Phi})(\bar{\xi})$ then there is a pair $(\gamma, T) \in \operatorname{Sat}_{\mathfrak{M}}(\Phi)$ such that $\bar{\xi} \models \gamma$.

Given the DAG representation of the PCTL formula $\Phi$, we follow the standard model checking procedure for CTL-like branching-time logics and compute $\operatorname{Sat}_{\mathfrak{M}}(\Psi)$ for the subformulas $\Psi$ assigned to the nodes in the DAG for $\Phi$ in a bottom-up manner. As the leaves of the DAG can be atomic propositions $a$ or the formula true, the base cases are $\operatorname{Sat}_{\mathfrak{M}}($ true $)=\{(\chi, S)\}$, and $\operatorname{Sat}_{\mathfrak{M}}(a)=\{(\chi,\{s \in S: a \in \mathscr{L}(s)\})\}$. Consider now the inner node $v$ of the DAG for $\Phi$ labelled by the outermost operator of the subformula $\Psi$. Suppose that the children of $v$ have already been treated, so when computing $\operatorname{Sat}_{\mathfrak{M}}(\Psi)$ the satisfaction sets of the proper subformulas of $\Psi$ are known. If $v$ is labelled by $\neg$ or $\wedge$, i. e., $\Psi=\neg \Psi^{\prime}$ or $\Psi=\Psi_{1} \wedge \Psi_{2}$, then 


$$
\begin{aligned}
& \operatorname{Sat}_{\mathfrak{M}}(\Psi)=\left\{(\gamma, S \backslash T):(\gamma, T) \in \operatorname{Sat}_{\mathfrak{M}}\left(\Psi^{\prime}\right)\right\} \text { respectively } \operatorname{Sat}_{\mathfrak{M}}(\Psi)=\left\{\left(\gamma_{1} \wedge \gamma_{2}, T_{1} \cap T_{2}\right):\left(\gamma_{i}, T_{i}\right) \in\right. \\
& \left.\operatorname{Sat}_{\mathfrak{M}}\left(\Psi_{i}\right), i=1,2\right\} . \text { If } \Psi=\mathbb{P}_{\bowtie c}\left(\Psi_{1} \cup \Psi_{2}\right), \text { then } \\
& \quad \operatorname{Sat}_{\mathfrak{M}}(\Psi)=\left\{\left(\gamma_{1} \wedge \gamma_{2} \wedge \delta_{\gamma_{1}, T_{1}, \gamma_{2}, T_{2}, R}\right):\left(\gamma_{1}, T_{1}\right) \in \operatorname{Sat}_{\mathfrak{M}}\left(\Psi_{1}\right),\left(\gamma_{2}, T_{2}\right) \in \operatorname{Sat}_{\mathfrak{M}}\left(\Psi_{2}\right), R \subseteq S\right\}
\end{aligned}
$$

where $\delta_{\gamma_{1}, T_{1}, \gamma_{2}, T_{2}, R}$ is the conjunction of the constraints $\operatorname{Pr}_{s}^{\mathfrak{M}}\left(T_{1} \cup T_{2}\right) \bowtie c$ for each state $s \in R$, and $\operatorname{Pr}_{s}^{\mathfrak{M}}\left(T_{1} \cup T_{2}\right) \not c$ for each state $s \in S \backslash R$. Here, $\operatorname{Pr}_{s}^{\mathfrak{M}}\left(T_{1} \cup T_{2}\right)$ is the rational function that has been computed using (i) a graph analysis to determine the set $U$ of states $s$ with $s \models \exists\left(T_{1} \cup T_{2}\right)$ and (ii) fractionfree Gaussian elimination (Section 3 ) to compute the rational functions $\operatorname{Pr}_{s}^{\mathfrak{N}}\left(\diamond T_{2}\right)$ in the pMC $\mathfrak{N}$ resulting from $\mathfrak{M}$ by turning the states in $(S \backslash U) \cup T_{2}$ into traps. If $f_{s}$ and $g_{s}$ are polynomials computed by fractionfree Gaussian elimination such that $\operatorname{Pr}_{s}^{\mathfrak{M}}\left(T_{1} \cup T_{2}\right)=f_{s} / g_{s}$ then $\operatorname{Pr}_{s}^{\mathfrak{M}}\left(T_{1} \cup T_{2}\right) \bowtie c$ is a shortform notation for $f_{s}-c \cdot g_{s} \bowtie 0$. The treatment of $\mathbb{P}_{\bowtie c}(\bigcirc \Psi)$ and the expectation operators is similar, and can be found in [16]. After treating a node of the DAG, we can simplify the set $\operatorname{Sat}_{\mathfrak{M}}(\Psi)$ by first removing all pairs $(\gamma, T)$ where $\gamma$ is not satisfiable (using algorithms for the existential theory of the reals), and afterwards combining all pairs with the same $T$-component, that is, instead of $m$ pairs $\left(\gamma_{1}, T\right), \ldots,\left(\gamma_{m}, T\right) \in \operatorname{Sat}_{\mathfrak{M}}(\Psi)$, we consider a single pair $\left(\gamma_{1} \vee \ldots \vee \gamma_{m}, T\right)$. To answer question (All), the algorithm finally returns the disjunction of all formulas $\gamma$ with $s_{\text {init }} \in T$ for $(\gamma, T) \in \operatorname{Sat}_{\mathfrak{M}}(\Phi)$.

Complexity bounds of (All) and (MC-E). The existential theory of the reals is known to be in PSPACE and NP-hard, and there is an upper bound on the time-complexity, namely $\ell^{k+1} \cdot d^{\mathscr{O}(k)}$ where $\ell$ is the number of constraints, $d$ the maximum degree of the polynomials in the constraints, and $k$ the number of parameters [3]. Recall from Section 3 that a known upper bound on the time-complexity of one-step fraction-free Gaussian elimination is $\mathscr{O}\left(\operatorname{poly}(n, d)^{k}\right)$, where $n$ is the number of equations, $d$ the maximum degree of the initial coefficient polynomials, and $k$ the number of parameters. Combining both approaches, the one-step fraction-free Gaussian elimination for solving linear equation systems with polynomial coefficients, and the existential theory of the reals for treating satisfiability of conjunctions of polynomial constraints, one directly obtains the following bound for the computational complexity of PCTL+EC model checking on augmented polynomial pMCs. Note that this assumes that the number of constraints in $\mathfrak{C}$ is at most polynomial in the size of $S$.

Theorem 4 (Exponential-time upper bound for problem (All)). Let $\Phi$ be a PCTL+EC formula. Given an augmented polynomial $p M C \mathfrak{M}$, where the maximum degree of transition probabilities $\mathbf{P}(s, t)$, and polynomials in the constraints in $\mathfrak{C}$ is $d$, a symbolic representation of the satisfaction function $\operatorname{Sat}_{\mathfrak{M}}(\Phi)$ is computable in time $\mathscr{O}\left(|\Phi| \cdot \operatorname{poly}(\operatorname{size}(\mathfrak{M}), d)^{k \cdot|\Phi|_{\mathbb{E}, \mathbb{C}}}\right)$, where $|\Phi|_{\mathbb{P}, \mathbb{E}, \mathbb{C}}$ is the number of probability, expectation and comparison operators in $\Phi$.

- Theorem 5 (PSPACE upper bound for problem (MC-E)). The existential PCTL+EC model checking problem (MC-E) for augmented $\mathrm{pMC}$ is in PSPACE.

Sketch of proof. The main idea of a polynomially space-bounded algorithm is to guess nondeterministically sets $T_{\Psi}$ of states for the subformulas $\Psi$ where the outermost operator is a probability, expectation or comparison operator, and then apply a polynomially space-bounded algorithm for the existential theory of the reals [3] to check whether there is a parameter valuation $\bar{\xi}$ such that $T_{\Psi}=\operatorname{Sat}_{\mathfrak{M}}(\Psi)(\bar{\xi})$ for all $\Psi$.

NP- and coNP-hardness of (MC-E) follow from results for IMCs [26, 6]. More precisely, [6] provides a polynomial reduction from SAT to the (existential and universal) PCTL model checking problem for IMCs. In fact, the reduction of [6] does not require full PCTL, instead Boolean combinations of simple probabilistic constraints $\mathbb{P}_{\geqslant c_{i}}\left(\bigcirc a_{i}\right)$ without nesting of the probability operators are sufficient. The following theorem strengthens this result by stating NP-hardness of (MC-E) even for formulas $\mathbb{P}_{>c}(\diamond a)$ consisting of a single probability constraint for a reachability condition. 
- Theorem 6 (NP-hardness for single probabilistic operator, multivariate case). Given an augmented polynomial pMC $\mathfrak{M}$ on parameters $\bar{x}$ with initial state $s_{\text {init }}$ and an atomic proposition a, and a probability threshold $c \in \mathbb{Q} \cap] 0,1\left[\right.$, the problem to decide whether there exists $\bar{\xi} \in X$ such that $\operatorname{Pr}_{s_{\text {init }}}^{\mathfrak{M}(\bar{\xi})}(\nabla a)>c$ is $N P$-hard, even for acyclic pMCs with the assigned transition probabilities being either constant, or linear in one parameter, i. e., $\mathbf{P}(s, t) \in \bigcup_{i=1}^{k} \mathbb{Q}\left[x_{i}\right], \operatorname{deg}(\mathbf{P}(s, t)) \leq 1$, for all $(s, t) \in E$, and where the polynomial constraints for the parameters $x_{1}, \ldots, x_{k}$ are of the form $f\left(x_{i}\right) \geqslant 0$ with $f \in \mathbb{Q}\left[x_{i}\right], \operatorname{deg}(f) \leq 2$.

Univariate pMCs. In many scenarios, the number of variables has a fixed bound instead of increasing with the model size. We consider here the case of univariate $\mathrm{pMC}$, i. e., pMC with a single parameter.

- Theorem 7 (PCTL+EC model checking without nesting in P, univariate case). Let $\Phi$ be a PCTL+EC formula without nested probability, expectation or comparison operators, and let $\mathfrak{M}$ be a polynomial pMC on the single parameter $x$. The problem to decide whether there exists an admissible parameter valuation $\xi \in X$ such that $\mathfrak{M}(\xi) \models \Phi$ is in $P$.

Sketch of proof. If we restrict PCTL+EC to Boolean combinations of probability, expectation, and comparison operators, (MC-E) can be dealt with by first computing polynomial constraints for $s_{\text {init }}$ for each probability, expectation, and comparison operator independently (this can be done in polynomial time by Lemma 2), and afterwards applying a polynomial-time algorithm for the univariate existential theory of the reals [4] once to the appropriate Boolean combination of the constraints.

- Theorem 8 (NP-completeness for full PCTL+EC, univariate case). Let $\Phi$ be a PCTL+EC formula, and let $\mathfrak{M}$ be a polynomial pMC on the single parameter $x$. The PCTL+EC model checking problem to decide whether there exists an admissible parameter valuation $\xi \in X$ such that $\mathfrak{M}(\xi) \models \Phi$ is $N P$ complete. NP-hardness even holds for acyclic polynomial pMCs and the fragment of PCTL+C that uses the comparison operator $\mathbb{C}_{\mathrm{Pr}}$, but not the probability operator $\mathbb{P}$, as well as for (cyclic) polynomial pMC in combination with PCTL.

(MC-E) for monotonic PCTL on univariate pMCs. The parameters in pMC typically have a fixed meaning, e.g., probability for the occurrence of an error, in which case the probability to reach a state where an error has occurred is increasing in $x$. This motivates the consideration of univariate pMCs and PCTL formulas that are monotonic in the following sense.

Given a univariate polynomial pMC $\mathfrak{M}=\left(S, s_{\text {init }}, E, \mathbf{P}\right)$, let $E_{+}$denote the set of edges $(s, t) \in E$ such that the polynomial $\mathbf{P}(s, t)$ is monotonically increasing in $X$, i. e., whenever $\xi_{1}, \xi_{2} \in X$ and $\xi_{1}<\xi_{2}$ then $\mathbf{P}(s, t)\left(\xi_{1}\right) \leqslant \mathbf{P}(s, t)\left(\xi_{2}\right)$. Let $S_{+}$denote the set of states $s$ such that for each finite path $\pi=s_{0} s_{1} \ldots s_{m}$ with $s_{m}=s$ we have $\left(s_{i}, s_{i+1}\right) \in E_{+}$for $i=0,1, \ldots, m-1$.

As $(s, t) \in E_{+}$iff there is no value $\xi \in \mathbb{R}$ such that $\xi=\chi \wedge\left(\mathbf{P}(s, t)^{\prime}<0\right)$, the set $E_{+}$is computable in polynomial time using a polynomial-time algorithm for the univariate theory of the reals [4]. Here, $\chi$ is as before the Boolean combination of polynomial constraints characterizing the set $X$ of admissible parameter values, and $\mathbf{P}(s, t)^{\prime}$ is the first derivative of the polynomial $\mathbf{P}(s, t)$. Thus, the set $S_{+}$is computable in polynomial time.

- Lemma 9. Let $\mathfrak{M}$ be a univariate polynomial pMC and $\Psi$ a monotonic PCTL formula, that is, $\Psi$ is in the PCTL fragment obtained by the following grammar:

$$
\begin{aligned}
& \Phi \quad:=a \in S_{+}|\Phi \wedge \Phi| \Phi \vee \Phi\left|\mathbb{P}_{\geqslant c}(\varphi)\right| \mathbb{P}_{>c}(\varphi) \\
& \varphi \quad::=\bigcirc \Phi|\Phi \cup \Phi| \Phi R \Phi|\diamond \Phi| \square \Phi
\end{aligned}
$$

where $c \in \mathbb{Q}_{>0}$. Then, $\operatorname{Sat}_{\mathfrak{M}\left(\xi_{1}\right)}(\Psi) \subseteq \operatorname{Sat}_{\mathfrak{M}\left(\xi_{2}\right)}(\Psi)$ for any two valuations $\xi_{1}$ and $\xi_{2}$ of $x$ with $\xi_{1}<\xi_{2}$. 
Hence, if $\Psi$ is monotonic then the satisfaction function $X \rightarrow 2^{S}, \xi \mapsto \operatorname{Sat}_{\mathfrak{M}}(\Psi)(\xi)=\operatorname{Sat}_{\mathfrak{M}(\xi)}(\Psi)$ is monotonic. For each monotonic PCTL formula $\Psi$ there exist $S_{\Psi} \subseteq S$ and $\xi_{\Psi} \in X$ such that $\operatorname{Sat}_{\mathfrak{M}(\xi)}(\Psi)=$ $S_{\Psi}$ for all $\xi \geqslant \xi_{\Psi}$ and $\operatorname{Sat}_{\mathfrak{M}\left(\xi^{\prime}\right)}(\Psi) \subseteq S_{\Psi}$ for all $\xi^{\prime}<\xi_{\Psi}$. To decide (MC-E) for a given monotonic formula $\Phi$, it suffices to determine the sets $S_{\Psi}$ for the sub-state formulas $\Psi$ of $\Phi$. This can be done in polynomial time. Using this observation, we obtain:

- Theorem 10 ((MC-E) for monotonic PCTL on univariate pMC). Let $\mathfrak{M}=\left(S, s_{\text {init }}, E, \mathbf{P}, \mathfrak{C}\right)$ be a univariate polynomial pMC on $x$, and $\Phi$ a monotonic PCTL formula. Then the model checking problem to decide whether there exists an admissible parameter valuation $\xi$ for $x$ such that $\mathfrak{M}(\xi) \models \Phi$ is in $P$.

Model checking PCTL+EC on MCs with parametric weights. We now consider the case where $\mathscr{M}$ is an ordinary Markov chain augmented with a parametric weight function $\operatorname{wgt}: S \rightarrow \mathbb{Q}[\bar{x}]$. Given a set $T \subseteq S$ such that $\operatorname{Pr}_{s}^{\mathscr{M}}(\diamond T)=1$ for all states $s \in S$, the vector of the expected accumulated weights $e=\left(\mathrm{E}_{s}^{\mathscr{M}}(\forall T)\right)_{s \in S}$ is computable as the unique solution of a linear equation system of the form $A \cdot e=b$, where the matrix $A$ is non-parametric, and only the vector $b$ depends on $\bar{x}$. By Lemma $3, \mathrm{E}_{s}^{\mathscr{M}}(\forall T)$ is a polynomial of the form $\sum_{t \in S} \beta_{s, t} \cdot \operatorname{wgt}(t)$ with $\beta_{s, t} \in \mathbb{Q}$ for all $s \in S$, and can be computed in polynomial time. The expected mean payoff for a given set $T$ is given by $\mathrm{E}_{s}^{\mathscr{M}}(\mathrm{mp}(T))=\sum_{\mathrm{BSCC} B} \operatorname{Pr}_{s}^{\mathscr{M}}(\diamond B) \cdot \operatorname{mp}(B)(T)$ where $\operatorname{mp}(B)(T)=\sum_{t \in T} \zeta_{t} \cdot w g t_{T}(t)$ with $\zeta_{t}$ being the steady-state probability for state $t$ inside $B$ (viewed as a strongly connected Markov chain), and $w g t_{T}(t)=0$ if $t \notin T, w g t_{T}(t)=w g t(t)$ for $t \in T$. As the transition probabilities are non-parametric, the steady-state probabilities are obtained as the unique solution of a non-parametric linear equation system. So both types of expectations can be computed in polynomial time. Unfortunately, the treatment of formulas with nested expectation operators is more involved. Using the standard computation scheme that processes the DAG-representation of the given PCTL+EC formula in a bottom-up manner to treat inner subformulas first, the combination of polynomial constraints after the consideration of an inner node is still as problematic as in the pMC-case. Using known algorithms for the existential theory of the reals yields the following bound.

- Theorem 11 (Time complexity of PCTL+EC model checking with parametric weights). Let $\mathscr{M}$ be an MC with parametric weights over $k$ parameters, and $\Phi$ a PCTL $+E C$ formula. The problem $(M C-E)$

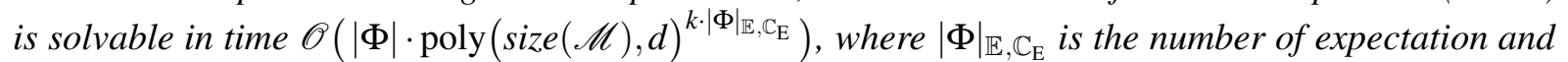
expectation comparison operators in the formula, and $d$ the maximum degree of the polynomials assigned as weights.

If there is only one parameter, the model checking for MCs with parametric weights is solvable in polynomial time for the fragment of PCTL+EC without nested formulas (cf. Theorem 7).

\section{Conclusion}

In this paper we revisited the model checking problem for $\mathrm{pMC}$ and PCTL-like formulas. The purpose of the first part is to draw attention to the fraction-free Gaussian elimination for computing rational functions for reachability probabilities, expected accumulated weights and expected mean payoffs as an alternative to the gcd-based algorithms that have been considered before and are known to suffer from the high complexity of gcd-computations for multivariate polynomials. The experiments with our (not yet optimized) implementation indicate that such an approach can indeed be feasible and beneficial in practice. We thus intend to refine this implementation in future work, including research into further structural heuristics and the potential of a combination with gcd-based simplifications at opportune moments.

In the second part of the paper we studied the complexity of the model checking problem for pMC and PCTL and its extension PCTL+EC by expectation and comparison operators. We identified instances 
where the model checking problem is NP-hard as well as fragments of PCTL+EC where the model checking problem is solvable in polynomial time. The latter includes the model checking problem for Boolean combinations of probability or expectation conditions for univariate pMCs. This result has been obtained using the fraction-free Gaussian elimination to compute rational functions for reachability probabilities or expected accumulated weights or expected mean payoffs, and polynomial time algorithms for the theory of the reals over a fixed number of variables. As the time complexity of the fraction-free Gaussian elimination is also polynomial for matrices and vectors with a fixed number of parameters and the polynomial-time decidability for the theory of the reals also holds when the number of variables is fixed [4], Theorem 7] also holds for pMC with a fixed number of parameters.

\section{References}

[1] Christel Baier \& Joost-Pieter Katoen (2008): Principles of Model Checking. The MIT Press.

[2] Erwin H. Bareiss (1972): Computational Solutions of Matrix Problems over an Integral Domain. IMA Journal of Applied Mathematics 10(1), pp. 68-104, doi 10.1093/imamat/10.1.68.

[3] Saugata Basu, Richard Pollack \& Marie-Françoise Roy (2008): Algorithms in Real Algebraic Geometry. Springer.

[4] Michael Ben-Or, Dexter Kozen \& John Reif (1986): The Complexity of Elementary Algebra and Geometry. Journal of Computer and System Sciences 32(2), pp. 251-264, doi:10.1016/0022-0000(86)90029-2.

[5] Michael Benedikt, Rastislav Lenhardt \& James Worrell (2013): LTL Model Checking of Interval Markov Chains. In: 19th Int. Conference on Tools and Algorithms for the Construction and Analysis of Systems (TACAS), LNCS 7795, Springer, pp. 32-46, doi:10.1007/978-3-642-36742-7_3.

[6] Krishnendu Chatterjee, Koushik Sen \& Thomas A. Henzinger (2008): Model-Checking omega-Regular Properties of Interval Markov Chains. In: 11th Int. Conference on Foundations of Software Science and Computational Structures (FoSSaCS), LNCS 4962, Springer, pp. 302-317, doi 10.1007/978-3-540-78499-9_22 .

[7] Frank Ciesinski, Christel Baier, Marcus Größer \& Joachim Klein (2008): Reduction Techniques for Model Checking Markov Decision Processes. In: 5th Int. Conference on Quantitative Evaluation of Systems (QEST), IEEE, pp. 45-54, doi:10.1109/QEST.2008.45.

[8] Conrado Daws (2005): Symbolic and Parametric Model Checking of Discrete-Time Markov Chains. In: 1st Int. Colloquium on Theoretical Aspects of Computing (ICTAC), LNCS 3407, Springer, pp. 280-294, doi: $10.1007 / 978-3-540-31862-0 \_21$.

[9] Christian Dehnert, Sebastian Junges, Nils Jansen, Florian Corzilius, Matthias Volk, Harold Bruintjes, JoostPieter Katoen \& Erika Ábrahám (2015): PROPhESY: A PRObabilistic ParamEter SYnthesis Tool. In: 27th Int. Conference on Computer Aided Verification (CAV), LNCS 9206, Springer, pp. 214-231, doi:10.1007/9783-319-21690-4_13.

[10] Christian Dehnert, Sebastian Junges, Joost-Pieter Katoen \& Matthias Volk (2017): A Storm is Coming: A Modern Probabilistic Model Checker. In: 29th Int. Conference on Computer Aided Verification (CAV), LNCS 10427, Springer, pp. 592-600, doi:10.1007/978-3-319-63390-9_31.

[11] Antonio Filieri, Carlo Ghezzi \& Giordano Tamburrelli (2011): Run-time efficient probabilistic model checking. In: 33rd Int. Conference on Software Engineering (ICSE), ACM, pp. 341-350, doi 10.1145/1985793.1985840

[12] Keith O. Geddes, Stephen R. Czapor \& George Labahn (1993): Algorithms for Computer Algebra. Kluwer.

[13] Ernst Moritz Hahn, Holger Hermanns, Björn Wachter \& Lijun Zhang (2010): PARAM: A Model Checker for Parametric Markov Models. In: 22nd Int. Conference on Computer Aided Verification (CAV), LNCS 6174, Springer, pp. 660-664, doi:10.1007/978-3-642-14295-6_56. 
[14] Ernst Moritz Hahn, Holger Hermanns \& Lijun Zhang (2011): Probabilistic reachability for parametric Markov models. Int. Journal on Software Tools for Technology Transfer 13(1), pp. 3-19, doi:10.1007/s10009-0100146-X

[15] Hans Hansson \& Bengt Jonsson (1994): A logic for reasoning about time and reliability. Formal Aspects of Computing 6(5), pp. 512-535, doi:10.1007/bf01211866.

[16] Lisa Hutschenreiter, Christel Baier \& Joachim Klein (2017): Parametric Markov Chains: PCTL Complexity and Fraction-free Gaussian Elimination (extended version). Available at http://wwwtcs . inf . tu-dresden. de/ALGI/PUB/GandALF17/

[17] Nils Jansen, Florian Corzilius, Matthias Volk, Ralf Wimmer, Erika Ábrahám, Joost-Pieter Katoen \& Bernd Becker (2014): Accelerating Parametric Probabilistic Verification. In: 11th Conference on Quantitative Evaluation of Systems (QEST), LNCS 8657, Springer, pp. 404-420, doi 10.1007/978-3-319-10696-0_31

[18] Bengt Jonsson \& Kim Guldstrand Larsen (1991): Specification and Refinement of Probabilistic Processes. In: 6th Annual Symposium on Logic in Computer Science (LICS), IEEE, pp. 266-277, doi $10.1109 /$ LICS.1991.151651.

[19] Ravindran Kannan (1985): Solving Systems of Linear Equations over Polynomials. Theoretical Computer Science 39, pp. 69-88, doi:10.1016/0304-3975(85)90131-8.

[20] Vidyadhar G. Kulkarni (1995): Modeling and analysis of stochastic systems. Chapman \& Hall.

[21] Marta Z. Kwiatkowska, Gethin Norman \& David Parker (2011): PRISM 4.0: Verification of Probabilistic Real-Time Systems. In: 23rd Int. Conference on Computer Aided Verification (CAV), LNCS 6806, Springer, pp. 585-591, doi:10.1007/978-3-642-22110-1_47.

[22] Ruggero Lanotte, Andrea Maggiolo-Schettini \& Angelo Troina (2007): Parametric probabilistic transition systems for system design and analysis. Formal Aspects of Computing 19(1), pp. 93-109, doi:10.1007/s00165006-0015-2.

[23] Michael T. McClellan (1973): The exact solution of systems of linear equations with polynomial coefficients. Journal of the Association for Computing Machinery 20(4), pp. 563-588, doi:10.1145/321784.321787

[24] George Nakos, Peter R. Turner \& Robert M. Williams (1997): Fraction-free algorithms for linear and polynomial equations. ACM SIGSAM Bulletin 31(3), pp. 11-19, doi $10.1145 / 271130.271133$.

[25] Tim Quatmann, Christian Dehnert, Nils Jansen, Sebastian Junges \& Joost-Pieter Katoen (2016): Parameter Synthesis for Markov Models: Faster Than Ever. In: 14th Int. Symposium on Automated Technology for Verification and Analysis (ATVA), LNCS 9938, Springer, pp. 50-67, doi 10.1007/978-3-319-46520-3_4.

[26] Koushik Sen, Mahesh Viswanathan \& Gul Agha (2006): Model-Checking Markov Chains in the Presence of Uncertainties. In: 12th Int. Conference on Tools and Algorithms for the Construction and Analysis of Systems (TACAS), LNCS 3920, Springer, pp. 394-410, doi:10.1007/11691372_26.

[27] William Y. Sit (1992): An Algorithm for Solving Parametric Linear Systems. Journal of Symbolic Computation 13(4), pp. 353-394, doi $10.1016 /$ S0747-7171(08)80104-6. 